BULL. AUSTRAL. MATH. SOC.

$05 B 25$

VOL. $23(1981), 313-315$.

\title{
SOME TRANSLATION PLANES CONSTRUCTED \\ BY MULTIPLE DERIVATION
}

\author{
N.L. JOHNSON
}

\begin{abstract}
It is noted that the translation planes of Rao and Rao may be constructed from a Desarguesian plane by the replacement of a set of disjoint derivable nets. Their plane of order 25 wich admits a collineation group splitting the infinite points into orbits of lengths 18 and 8 may be obtained by replacing exactly three disjoint derivable nets and may be viewed as being derived from the André nearfield plane of order 25 .
\end{abstract}

In [4], Rao and Rao construct a class of translation planes in the following manner:

Let $\Sigma$ denote the Desarguesian affine plane of order $q^{2}$ coordinatized by $\operatorname{GF}\left(q^{2}\right)$. $\Sigma$ may be identified with $K=\operatorname{GF}\left(q^{4}\right)$ where the components of $\Sigma$ are $a \mathrm{GF}\left(q^{2}\right)=a F$ where $a \in K$. Now let $V$ be a 2-dimensional subspace of $K$ over $G F(q)$ which is not of the form $a \mathrm{GF}\left(q^{2}\right)$. Rao and Rao [5] construct translation planes by taking images of such 2-spaces $V$ under the mapping $T: x \rightarrow x \alpha^{q^{2}+1}$ where $\alpha$ is a primitive root of $K$ and then determining certain situations under which the image sets are pairwise disjoint. (Notice that $V$ has exactly $q+1$ images under $\langle T\rangle$.

The mapping $T$ acts as a collineation of $\Sigma$ which fixes each component and will induce a collineation in the planes constructed above

Received 19 November 1980. 
but $T$ will clearly act differently on the new planes.

NOTE 1. Every 2-dimensional subspace of $K$ over $\mathrm{GF}(g)$ is either a component (line) of $\Sigma$ or a Baer subplane of $\Sigma$.

Proof. See, for example, (3.8) [3].

NOTE 2. The $q+1$ images of $V$ (as Baer subplanes of $\Sigma$ ) under $T$ must have the same infinite points in $\Sigma$. Thus $\left\{V T^{i}\right\}$ is the set of $q+1$ Baer subplanes of a derivable net in $\Sigma$.

Let $V, \bar{U}$ be distinct 2-spaces which are not components of $\Sigma$. The planes of Rao and Rao are obtained by assuming sets $\left\{V T^{i}\right\}$ and $\left\{\bar{U} T^{i}\right\}$ are disjoint. So:

NOTE 3. The planes constructed by Rao and Rao are the planes obtained from $\Sigma$ by the replacement of a set of pairwise disjoint derivable nets in $\Sigma$.

NOTE 4. Corollary (2.11) [5] shows that two such nets can always be found which are disjoint. By Bruck [1] (7.6), the corresponding planes are Andre planes.

In [6] Rao and Rao completely determine the full collineation group of a plane of order 25 constructed as above and which admits a collineation group that has infinite point orbits of lengths 8 and 18 .

In [4] Johnson and Ostrom considered translation planes admitting several homology centers and constructed a class of planes admitting homologies of order 3 with exactly eight centers all of which are in the same orbit (see [4] (2.8)). The plane constructed in [4] of order 25 is of particular interest. It not only has the eight homology centers in an orbit but also has 18 homologies of order 2 whose centers are in a single orbit.

The full collineation group $G$ of the planes constructed by Johnson and Ostrom is determined by Johnson in [2] and $G \supseteq \operatorname{PGL}(2,3)$ by $(2.6)$ [4].

NOTE 5. The plane of Rao and Rao of order 25 constructed in [6] is the plane of Johnson and Ostrom [4]. It may be constructed by the derivation of the Andre nearfield plane of order 25 and is the unique 
plane of Bruck type \{1\}.

Proof. There are only $5-1=4$ possible pairwise disjoint derivable nets (with 6 components) in the Desarguesian plane $\Sigma$ of order 25. By Note 3 the Rao and Rao plane must be so constructed. If it is obtained by replacement of one or two nets, the plane is Hall or André and cannot have the orbit structure that it has. Thus, it must have taken three nets to construct the plane. Thus, the plane is either Hall or the triple of nets is nonlinear in the sense of Bruck [1]. By Bruck [1] (7.67), there is a unique such plane (also see [4], Section 3 ).

\section{References}

[1] R.H. Bruck, "Construction problems of finite projective planes", Combinatorial mathematics and its applications, 426-514 (Proc. Conf. Univ. North Carolina, Chapel Hill, 1967. University of North Carolina Press, Chapel Hill, 1969).

[2] N.L. Johnson, "The translation planes of Bruck type $\{1\}$ ", Arch. Math. (Basel) 26 (1975), 554-560.

[3] N.L. Johnson, "Derivation in infinite planes", Pacific J. Math. 43 (1972), 387-402.

[4] N.L. Johnson and T.G. Ostrom, "Translation planes with several homology or elation groups of order 3 ", Geom. Dedicata 2 (1973-1974), 65-81.

[5] M.L. Narayana Rao and K. Kuppuswamy Rao, "A class of non-Desarguesian planes", J. Combin. Theory Ser. A 19 (1975), 247-255.

[6] M.L. Narayana Rao and K. Kuppuswamy Rao, "A translation plane of order 25 and its full collineation group", Bull. Austral. Math. Soc. 19 (1978), 351-362.

\footnotetext{
Department of Mathematics, University of lowa, lowa City, lowa 52242, USA.
} 